\title{
Perceived Differences of Lightness Deviation during the Appearance of Simultaneous Contrast Effect in Printing Process
}

\author{
Martina HAJDEK, Krunoslav HAJDEK, Ivan BUDIMIR
}

\begin{abstract}
This paper shows the research data of psychophysical visual effect of simultaneous contrast when changing RTV of background lightness in the printing process An experimental research on graphic reproductions made in the offset printing technique with specifically constructed design solutions that cause the above mentioned effect was carried out. Samples were designed as ten various lightness variances of background, with a range from $10 \%$ RTV to $100 \%$ RTV, on which there are primary stimuli with value of $50 \%$ RTV. The visual experiment was performed by using simultaneous binocular harmonization technique, and it involved 20 respondents. The respondents were tasked with harmonizing the primary stimuli from the test reproduction in relation to the referential one, so that they were to be perceived as equal on both reproductions. For statistical data processing, one-way repeated measurements ANOVA was used, which found statistically consequential differences among means of deviations in perceived lightness of primary stimuli. Post-hoc analysis by Fisher shows pairs that are reciprocally different $(p<0,05)$. The obtained results clearly indicate the regularities of the appearance of simultaneous contrast effect on printed medium, all with the aim of creating a model for predicting it and finding a conceptual design solution in which the effect is pronounced the least.
\end{abstract}

Keywords: ANOVA; print; reproduction; simultaneous contrast

\section{INTRODUCTION}

The simultaneous contrast effect, which is better known as the induction effect, is one of the three background psychophysical visual effects. Any background psychophysical visual effect is in its essence an undesirable effect due to the fact that it changes the perception of the observed color/lightness of the observer, which then results in a distorted perception of the quantitative and qualitative attributes of the color/lightness on the observed image. One of the main goals of graphic production in terms of printing is to produce reproductions as similar as possible to the original, and the presence of researched psychophysical effects greatly affects this. Researched effect of simultaneous contrast was first discovered in 1810 by Von Goethe [1], and it was described by Chevreul in 1839 [2].

It is worth mentioning that the effect of crispening is very similar to the effect presented in this paper. It is significantly "younger" than the simultaneous contrast effect and it was discovered in relation to the simultaneous contrast effect not so long ago, in 1966, by a Japanese researcher Takasaki, who described it in his works [3, 4]. Even though both of these effects are background effects and are very similar. In contrast, effect of crispening occurs when intensity of the perceived color/lightness increases between the two primary stimuli which are mutually compared [5]. The occurrence of the color perceived in the human eye is actually a combination of various conditions and factors [6].

The effect studied in this paper (induction) is very interesting and often explored in a wide range of scientific areas. In painting, the renowned painter Van Gogh achieved the simultaneous contrast effect by using complementary pairs of colors in his works [7]. The induction effect has the strongest appearance with the combinations of complementary color pairs - chromatic simultaneous contrast [8]. Moreover, in their work, the authors Chen et al. describe in which way simultaneous contrast can be shown through artwork [9]. The authors
Gonzalez and Powers even research the influence of the effect on goldfish [10].

By using computers more extensively for the purpose of exploring the simultaneous contrast effect, there has been a much wider exploration of the occurrence of the effect, and thus, more sophisticated theories about the light that is needed for its manifestation have emerged [11-13]. Such models gave insight into a much stronger display of light constancy, but they never understand how big is lightness influence at induction [11]. In the last fifteen years, there has been an increasing interest in exploring the perceptual aspects of color manifestation in computer graphics [14].

Therefore, when presenting objects in a threedimensional space, while the perception of the displayed colors is constant, the objects will have a different color and the colors will be perceived as constant at different lighting conditions, with certain exceptions; and when the object is displayed in a two-dimensional space, for example on a computer screen, it is likely that many of the effects of color interaction will be different from those in a three-dimensional world [15]. In their paper, Wu et al. are concerned with the research on the differences and similarities between the cathode tubes and surface dyes [16]. Furthermore, Soranzo and Agostini explore the intersection between an illumination and a reflectance edge on CRT [17]. When viewing objects in a three-dimensional space, some of the boundaries of the observed objects are fragmented due to the partial occlusion or poor contrast between the shape and the background [18]. In his paper, Vladusich explores the psychophysical data that question the assumption embedded in many models of achromatic color perceptions - that achromatic colors are represented as points in a one-dimensional (1D) perceptual space or the absolute color spectrum, and he presents an alternative model in which the achromatic spectrum of colors that corresponds to the target area is relatively determined considering the surrounding light. Furthermore, he also proposed a new model of geometric approach to simultaneous contrast and achromatic color matching in terms of the vector sum of local lightness and contrast 
components. Moreover, he set the phase for a unique computer theory of achromatic color perception [19]. The authors Zavagno et al. research the role of physical lighting in the perception of lightness in simultaneous contrast [20].

The occurrence of this effect has recently also been explored in virtual reality, which is why in certain works there are two interpretations by means of systematically manipulating the viewing distance and the horizontal distance between the backgrounds of both the articulated and plain SLC display [21]. Furthermore, Menshikova researches the problem of lightness perception between two current models - the albedo hypothesis and the coplanar-ratio hypothesis on 3D images using a virtualreality technique [22].

In their work, Hajdek et al. explore influence of induction effect at achromatic and desaturated reproductions in relation to reproductions made in offset printing - color atlas made in the digital print, where the power of the effect depends on type of reproduction [23]. Furthermore, authors Hajdek et al. also examine strength of induction effect in relation to the reproductions made in the technique of offset printing - color atlas made in the technique of digital printing [24]. In the work by Hajdek et al., the power of induction effect in the digital medium is evaluated on a desaturated design solution in which the effect occurs, and which is displayed on two different computer screens [25].

The research presented in this paper is a continuation of a previous research carried out by Hajdek et al. in which the strength of the induction effect is researched in dependence of the different lightness of secondary stimuli in the achromatic design solution due to which the effect appears. The evaluation of the effect was carried out in relation to the reproductions made in the offset printing technique [26].

At all previous research of the effect of simultaneous contrast, it has not yet been explored with which intensity the effect of simultaneous contrast manifests itself in the offset printing technique on a concrete example in real production. Hence, in this paper, we will examine and present the intensity of the simultaneous contrast effect in relation to a reproduction made in the offset printing technique according to the CIP3 values and a reproduction made in the same printing technique by means of the tuning method [27, 28], and which the respondents will have the task to make in a way that the primary stimuli on their reproduction are perceived the same as the primary stimuli on reproduction that is made with the help of CIP3.

\section{EXPERIMENTAL PART}

The created design for the examination of the simultaneous contrast is made as one color (black) stimuli which triggers appearance of induction effect. The sample consists of a primary stimulus (internal field) that had RTV $50 \%$ of key color, and secondary stimuli (external field) with a value in the range from $10 \%$ RTV - $100 \%$ RTV of key color in magnification of each subsequent stimuli by 10\% RTV (Fig. 1).

On the printed/test form, the primary stimuli are arranged in such a way that each stimulus is located directly above one ink zone, while "blank" ink zone is set among stimuli, all with the aim of preventing the influence of adjacent zones on the coloration of the evaluated stimuli. In the above described manner of the form design of 24 existing zones on offset machine that has max. format of $51 \times 72 \mathrm{~cm}$, each second zone is blank, and vertical lines are multiplied up until the end of sheet (Fig. 2).

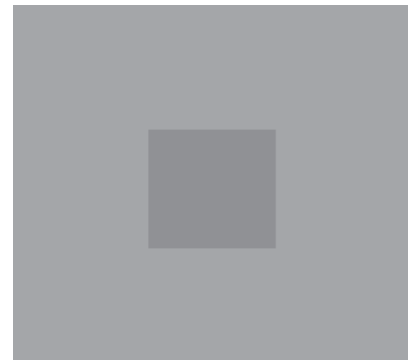

Figure 1 Layout of one primary and one secondary stimuli for the evaluation of the effect (primary stimuli of 50\% RTV, secondary stimuli of $40 \%$ RTV)

\begin{tabular}{|c|c|c|c|c|c|c|c|c|c|}
\hline - & $=$ & $=$ & $=$ & = & - & D & [ & [ & D \\
\hline i & i & i & $\mathbf{a}$ & $\bar{a}$ & $i$ & $i$ & $\bar{a}$ & $\overline{\mathbf{a}}$ & E \\
\hline i & $i$ & $i$ & $\overline{\mathbf{i}}$ & $\mathbf{i}$ & 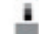 & $i$ & $\overline{\mathbf{a}}$ & $\bar{i}$ & $\bar{i}$ \\
\hline i & i & $i$ & $i$ & $\overline{\mathbf{i}}$ & i & $i$ & $\bar{i}$ & $\overline{\mathbf{i}}$ & $\dot{\theta}$ \\
\hline & 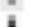 & 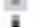 & 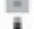 & 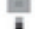 & $i$ & 뭉 & 5 & $\square$ & \\
\hline 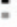 & 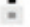 & $=$ & $=$ & 뭉 & & 0 & 무요 & $\dot{0}$ & 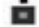 \\
\hline$=$ & $=$ & $=$ & $=$ & 무 & & D & $\mathbf{D}$ & $\mathbf{q}$ & 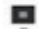 \\
\hline I & $i$ & $i$ & $i$ & i & $i$ & $i$ & i & 尚 & i \\
\hline i & i & i & i & $\overline{\mathbf{i}}$ & i & $i$ & $\overline{\mathbf{i}}$ & $\overline{\mathbf{a}}$ & 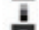 \\
\hline i & i & i & $\bar{i}$ & $\bar{i}$ & $\mathbf{i}$ & $i$ & $\bar{i}$ & i & 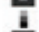 \\
\hline & & $=$ & $=$ & $=$ & & & & $=$ & \\
\hline 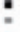 & - & 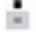 & 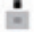 & 送 & 1 & i & a & 尚 & D \\
\hline $\mathbf{z}$ & i & i & i & $\overline{\mathbf{z}}$ & i & $i$ & i & i & 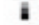 \\
\hline
\end{tabular}

Figure 2 Test form for the induction effect testing

For the visual evaluation of samples, the simultaneous binocular harmonization technique was selected [29] for comparison of primary stimuli on the referent reproduction and of primary stimuli obtained through the tuning method in the printing process. Twenty male participants, who participated in this research, were given the instructions to harmonize primary stimuli on the reproduction they were making (tuning) with primary stimuli on referential reproduction so that the primary stimuli from their reproduction were to be perceived as visually the same as the primary stimuli on the referential reproduction. On all reproductions obtained through the tuning method (20 respondents-20 reproductions), each primary stimuli was measured ten times in order to obtain the Lab mean of tuned areas on imprint which are taken into account when further measurements were obtained. On the reproduction obtained through CIP3 values, the arithmetic mean used for further measurements was also measured.

The evaluation process was performed as follows: first, the reproduction done according to the CIP3 values which was used as the referential one was printed, then each respondent began with the tuning of the reproduction (the ink tray was reversed and returned to its zero state) in which the primary stimuli were to be visually the same as the primary stimuli on the referential reproduction. After all respondents completed the "tuning" of the primary stimuli in relation to the referential and test reproduction, the values of both types of reproductions are calculated with the spectrophotometer usage (each primary stimulus was measured ten times).

The making of the test form was done in the offset printing technique with the help of the calibrated offset 
machine SM 74-5-P using the perceptual ICC rendering method in Photoshop. As a conversion option, the Adobe (ACE) option was selected, and for printing substrate the woodfree uncoated paper $\left(140 \mathrm{~g} / \mathrm{m}^{2}\right)$ was selected. Ambient visual conditions of the visual evaluation space were compliant with the ISO 3664:2009 guidelines D50 light source.

Printing form through which the reproductions were made was using Kodak CTP machine, the plate was Electra $\mathrm{XD}$ and the plate size was $745 \times 605 \mathrm{~cm}$. For plate production, the Magnus Q800 Platesetter by the same producer was used. For measuring Lab values, the X Rite i1 Pro spectrophotometer.

After tuning the CIE Lab values of primary stimuli on both reproductions were measured, along with the $\Delta L_{00}$, $\Delta E_{00}, \Delta C_{00}$ and $\Delta H_{00}$.

The $\Delta E_{00}$ formula helped calculate the research data of Lab values:

$$
\begin{aligned}
& \Delta L^{\prime}=L_{2}^{\prime}-L_{1}^{\prime} \\
& \Delta C_{a b}^{\prime}=C_{a b, b}^{\prime}-C_{a b, s}^{\prime} \\
& \Delta H_{a b}^{\prime}=\left[2\left(C_{a b, b}^{\prime} \cdot C_{a b, s}^{\prime}\right)^{0,5} \cdot \sin \left(\frac{\Delta C_{a b}^{\prime}}{2}\right)\right] \\
& \Delta E_{00}=\sqrt{\left(\frac{\Delta L^{\prime}}{k_{\mathrm{L}} S_{\mathrm{L}}}\right)^{2}+\left(\frac{\Delta C^{\prime}}{k_{\mathrm{C}} S_{\mathrm{C}}}\right)^{2}+\left(\frac{\Delta H^{\prime}}{k_{\mathrm{H}} S_{\mathrm{H}}}\right)^{2}+R_{\mathrm{T}}\left(\frac{\Delta C_{a b}^{\prime}}{k_{\mathrm{C}} S_{\mathrm{C}}}\right)^{2}\left(\frac{\Delta H_{a b}^{\prime}}{k_{\mathrm{H}} S_{\mathrm{H}}}\right)}
\end{aligned}
$$

\section{RESULTS OF RESEARCH}

The research results of primary stimulus on a design solution which causes the simultaneous contrast effect, shows them for different variations in the lightness of the secondary stimuli. Secondary stimuli had RTV values from $10 \%$ - $100 \%$ with magnification of each stimuli that follows by $10 \%$ RTV. Tab. 1 shows the measured arithmetic means of arithmetic Lab values of the primary stimuli on all ten different variations of the secondary stimuli.

Table 1 Lab data of means for "referential" primary stimuli of 50\% RTV on ten variations of secondary stimuli

\begin{tabular}{|c|c|c|c|}
\hline Background (RTV) & $L$ & $a$ & $b$ \\
\hline $10 \%$ & 61,33 & 1,20 & $-3,91$ \\
\hline $20 \%$ & 61,01 & 1,20 & $-3,91$ \\
\hline $30 \%$ & 60,81 & 1,20 & $-3,90$ \\
\hline $40 \%$ & 60,52 & 1,20 & $-3,90$ \\
\hline $50 \%$ & 60,03 & 1,20 & $-3,92$ \\
\hline $60 \%$ & 59,84 & 1,20 & $-3,92$ \\
\hline $70 \%$ & 59,33 & 1,20 & $-3,91$ \\
\hline $80 \%$ & 58,92 & 1,20 & $-3,91$ \\
\hline $90 \%$ & 58,64 & 1,20 & $-3,81$ \\
\hline $100 \%$ & 58,30 & 1,20 & $-3,81$ \\
\hline
\end{tabular}

The values shown in Tab. 1 point to slight deviation among values of Lab at referential primary stimuli which are unavoidable because of construction of offset machine, precisely because of the secondary stimuli which surround the primary stimuli. Due to the design solution which causes the researched effect to appear, and which appears on the printed form as if the primary stimuli and the secondary stimuli are located in the same coloration zone. When an operator on a machine wants to increase or decrease the application of color on a primary stimulus in order to perceive the stimulus as darker or brighter, she automatically increases or decreases the application of color on the secondary stimulus.

Consequently, during the measurement of the Lab value of the referential primary stimulus for the effect of simultaneous contrast, a tolerance of $\leq 4$ for the measured values of the referential primary stimulus was determined.

Tab. 2 shows values of Lab means at "tuned" primary stimuli.

Table 2 Lab values of means of "tuned" primary stimuli of $50 \%$ RTV on ten variations of secondary stimuli

\begin{tabular}{|c|c|c|c|}
\hline Background (RTV) & $L$ & $a$ & $b$ \\
\hline $10 \%$ & 58,71 & 1,01 & $-3,72$ \\
\hline $20 \%$ & 58,33 & 1,00 & $-3,74$ \\
\hline $30 \%$ & 58,22 & 1,11 & $-3,72$ \\
\hline $40 \%$ & 58,20 & 1,12 & $-3,90$ \\
\hline $50 \%$ & 57,84 & 1,13 & $-4,01$ \\
\hline $60 \%$ & 62,11 & 1,23 & $-3,94$ \\
\hline $70 \%$ & 62,62 & 1,30 & $-4,01$ \\
\hline $80 \%$ & 62,72 & 1,31 & $-4,01$ \\
\hline $90 \%$ & 62,80 & 1,32 & $-4,02$ \\
\hline $100 \%$ & 62,93 & 1,30 & $-4,03$ \\
\hline
\end{tabular}

Tab. 3 shows the measured values of $\Delta E_{00}, \Delta L_{00}, \Delta C_{00}$ and $\Delta H_{00}$ between the referential primary stimuli on a reproduction made in accordance with the CIP3 values and the arithmetic mean of the "tuned" primary stimuli on a reproduction made using the tuning method that is identical to the printing process. The obtained values of $\Delta L_{00}$ are presented as positive and negative in the examined effect, depending on whether the tuned field of the primary stimulus is darker or lighter than the referential primary stimulus.

Table 3 Overview of calculated measurements among evaluated primary stimuli (CIP3-tuning method)

\begin{tabular}{|c|c|c|c|c|}
\hline Background (RTV) & $\Delta E_{00}$ & $\Delta L_{00}$ & $\Delta C_{00}$ & $\Delta H_{00}$ \\
\hline $10 \%$ & 2,312 & 2,292 & 0,264 & 0,192 \\
\hline $20 \%$ & 2,414 & 2,395 & 0,262 & 0,194 \\
\hline $30 \%$ & 2,321 & 2,304 & 0,214 & 0,055 \\
\hline $40 \%$ & 2,054 & 2,045 & 0,053 & 0,133 \\
\hline $50 \%$ & 1,995 & 1,973 & $-0,030$ & $-0,025$ \\
\hline $60 \%$ & 2,003 & $-2,002$ & 0,002 & 0,002 \\
\hline $70 \%$ & 2,872 & $-2,860$ & $-0,134$ & $-0,094$ \\
\hline $80 \%$ & 3,314 & $-3,314$ & $-0,133$ & $-0,092$ \\
\hline $90 \%$ & 3,663 & $-3,662$ & $-0,211$ & $-0,050$ \\
\hline $100 \%$ & 4,020 & $-4,014$ & $-0,210$ & $-0,052$ \\
\hline
\end{tabular}

\section{DISCUSSION OF RESULTS}

Based on the psychophysical visual experiment, a statistical analysis of the collected data was done. Values in lightness deviations among physical values and perceived of primary stimuli were analyzed. The mentioned deviations in lightness were calculated for the primary stimuli of 50\% RTV. The measure of lightness deviation is defined as the arithmetic mean of the deviation observed by the respondents. STATISTICA 12 was used for statistical analysis. Data analysis combines the measurements of descriptive parameters in lightness deviations, Box \& Whisker graphical representation of the results, results of K-S test that includes ANOVA results 
and Fisher's post-hoc analysis, which brought to an end the identification of differences between the means.

\subsection{Descriptive Statistics of Lightness Differences}

Tab. 4 shows the results of the descriptive statistical analysis for lightness differences. The deviations in lightness were measured for the primary stimulus on all 10 variations of the secondary stimuli with an increment of $10 \%$ RTV for each stimulus that follows.

Table 4 Descriptive analysis in perceived lightness deviations of primary stimuli of $50 \%$ RTV

\begin{tabular}{|c|c|c|c|c|c|}
\hline \multirow{2}{*}{$\begin{array}{c}\text { Background } \\
(\mathrm{RTV})\end{array}$} & \multicolumn{5}{|c|}{ Data of lightness deviations } \\
\cline { 2 - 6 } & Mean \pm St.dev. & Median & Minimum & Maximum & Variance \\
\hline $10 \%$ & $2,360 \pm 3,660$ & 2,291 & $-2,460$ & 7,412 & 13,424 \\
\hline $20 \%$ & $2,511 \pm 4,201$ & 2,392 & $-3,462$ & 8,830 & 17,610 \\
\hline $30 \%$ & $2,463 \pm 4,642$ & 2,311 & $-4,691$ & 10,174 & 21,533 \\
\hline $40 \%$ & $2,110 \pm 3,203$ & 2,040 & $-2,484$ & 6,923 & 10,254 \\
\hline $50 \%$ & $2,064 \pm 3,600$ & 1,972 & $-2,923$ & 7,260 & 12,952 \\
\hline $60 \%$ & $-1,923 \pm 3,352$ & $-2,001$ & $-7,704$ & 4,290 & 11,250 \\
\hline $70 \%$ & $-2,782 \pm 3,404$ & $-2,863$ & $-7,680$ & 2,352 & 11,573 \\
\hline $80 \%$ & $-3,121 \pm 5,103$ & $-3,294$ & $-10,864$ & 5,312 & 25,992 \\
\hline $90 \%$ & $-3,433 \pm 5,382$ & $-3,652$ & $-11,131$ & 4,851 & 28,914 \\
\hline $100 \%$ & $-3,780 \pm 5,611$ & $-4,011$ & $-11,612$ & 4,683 & 31,432 \\
\hline
\end{tabular}

From Tab. 4, it can be seen that the conducted descriptive statistics indicate that in terms of the calculated data of St. dev. and var., the differences in lightness are significant. In terms of the results between the minimum and the maximum, it can be seen that the ranges are very pronounced, and that the median values are almost identical to the arithmetic means.

Fig. 3 presents the Box \& Whisker graphical overview of arithmetic means in lightness.

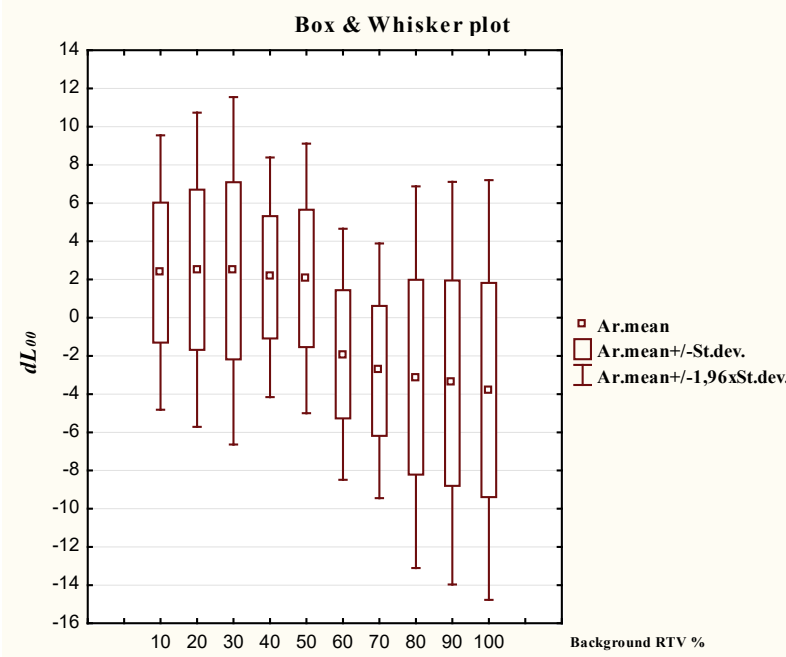

Figure 3 Box \& Whisker graphical overview of measured deviations of $\Delta L_{00}$ lightness differences for the primary stimulus of $50 \%$ RTV on 10 variations of secondary stimulus

\subsection{Analysis of the Variance of Repeated Measurements (ANOVA)}

In this chapter, the compliance of the collected data with the normal distribution law was examined.

Since almost all variables are in compliance with the normal distribution law (Tab. 5), the next step is to conduct the ANOVA analysis with repeated measurements. ANOVA measurements are shown in Tab. 6 all with the aim of determining the difference in strength of induction effect depending on different variations in lightness of the secondary stimuli.

Table 5 Overview of the results of K-S test for lightness difference in the primary stimuli of $50 \%$ RTV

\begin{tabular}{|c|c|c|}
\hline Background (RTV) & $\operatorname{Max} D$ & $\mathrm{~K}-\mathrm{S} p$ \\
\hline $10 \%$ & 0,141 & $p>0,20$ \\
\hline $20 \%$ & 0,122 & $p>0,20$ \\
\hline $30 \%$ & 0,110 & $p>0,20$ \\
\hline $40 \%$ & 0,110 & $p>0,20$ \\
\hline $50 \%$ & 0,132 & $p>0,20$ \\
\hline $60 \%$ & 0,090 & $p>0,20$ \\
\hline $70 \%$ & 0,112 & $p>0,20$ \\
\hline $80 \%$ & 0,124 & $p>0,20$ \\
\hline $90 \%$ & 0,091 & $p>0,20$ \\
\hline $100 \%$ & 0,120 & $p>0,20$ \\
\hline
\end{tabular}

Legend: Max $D$ statistics, K-S p-empirical $p$-value

Table 6 Overview of results of the ANOVA analysis of the lightness difference in the primary stimulus of $50 \%$ RTV- (repeated measurements)

\begin{tabular}{|c|c|c|c|c|c|}
\hline Effect & $S S$ & Freedom degrees & $M S$ & $F$ & $p$ \\
\hline R1 & 725,784 & 9 & 80,642 & 4,441 & 0,000 \\
\hline Error & 1470,602 & 81 & 18,163 & & \\
\hline
\end{tabular}

From Tab. 6, it can be seen that it has been concluded that $F=4,441$ with the statistical consequential of $F$-test being $p=0,00<0,05$. So, this determined that in this research data there are statistically significant differences among the means in results.

Afterwards, the Fisher post-hoc analysis (Tab. 7) is carried out, all with the aim of determining the pairs that have means that are statistically significantly different.

It is evident from Tab. 7 that according to the obtained data made with Fishers post-hoc analysis it has been shown that means of lightness differences $\left(\Delta L_{00}\right)$ in the primary stimuli where the values of RTV background go from $10 \%$ - 50\% have no statistically significantly differences. Furthermore, there are no statistically significant differences in primary stimuli where values of RTV background are from $60 \%$ - 100\%. Highest intensity of induction was achieved in primary stimuli that have value of the background of $100 \%$ RTV. In the mentioned sample, the mean of lightness difference (Tab. 4) is $\mu_{100}=-3,78$ while in the same sample, the median reaches the value of $\operatorname{Med}_{100}=-4,01$. A slightly weaker intensity of the effect was achieved in the primary stimuli that have values of the background of $70 \%, 80 \%$ and $90 \%$ RTV with the arithmetic mean differences in lightness of $\mu_{80}=-3,12$ and $\mu_{90}=-3,43$ and medians of $\operatorname{Med}_{80}=-3,29$ and $\operatorname{Med}_{90}=$ $-3,65$. The mean of the difference in the primary stimuli that has value of the background $70 \% \mathrm{RTV}$ is $\mu_{70}=-2,78$ while the median is $\mathrm{Med}_{70}=-2,86$.

In the primary stimuli that have values of the RTV background from $10 \%-50 \%$, a shift in occurrence of lightness in opposite direction was observed. At the value of secondary stimuli of $10 \% \mathrm{RTV}$, the mean of the difference in lightness is $\mu_{10}=2,36$ while the median is $\operatorname{Med}_{10}=2,29$. At the value of the secondary stimulus of $20 \% \mathrm{RTV}$, the mean of lightness shift is $\mu_{20}=2,51$ while the value of the median is $\operatorname{Med}_{20}=2,39$ and at the value of the secondary stimulus of $30 \% \mathrm{RTV}$, the primary stimulus has an average intensity of $\mu_{30}=2,46$ and its median is $\operatorname{Med}_{30}=2,31$. Primary stimulus that has value of the RTV background $40 \%$, the arithmetic mean of the lightness difference is $\mu_{40}=2,11$ and the median is $\operatorname{Med}_{40}=2,04$ 
while in the secondary stimulus with the value of $50 \%$ RTV, the mean of lightness shift is $\mu_{50}=2,06$ and the median is $\operatorname{Med}_{50}=1,97$.

From the results presented above, it is noted that the respondents, when tuning the test primary stimuli compared to the referential (CIP3) in the RTV values of background $10 \%-50 \%$, tuned primary stimuli on test reproduction so that they are perceived as darker than primary stimuli on referential reproduction.

Table 7 Overview of results of the Fisher post hoc analysis for primary stimuli 50\% RTV

\begin{tabular}{|c|c|c|c|c|c|c|c|c|c|}
\hline \multirow{2}{*}{$\begin{array}{l}\text { Background } \\
\text { (RTV) }\end{array}$} & \multicolumn{9}{|c|}{ Probabilities of the post hoc analysis } \\
\hline & $10 \%$ & $20 \%$ & $30 \%$ & $40 \%$ & $50 \%$ & $60 \%$ & $70 \%$ & $80 \%$ & $90 \%$ \\
\hline $10 \%$ & - & & & & & & & & \\
\hline $20 \%$ & 0,938 & - & & & & & & & \\
\hline $30 \%$ & 0,961 & 0,976 & - & & & & & & \\
\hline $40 \%$ & 0,896 & 0,835 & 0,858 & - & & & & & \\
\hline $50 \%$ & 0,872 & 0,812 & 0,835 & 0,976 & - & & & & \\
\hline $60 \%$ & 0,027 & 0,022 & 0,024 & 0,037 & 0,040 & - & & & \\
\hline $70 \%$ & 0,008 & 0,006 & 0,007 & 0,012 & 0,013 & 0,651 & - & & \\
\hline $80 \%$ & 0,005 & 0,004 & 0,004 & 0,007 & 0,008 & 0,530 & 0,860 & - & \\
\hline $90 \%$ & 0,003 & 0,002 & 0,002 & 0,004 & 0,005 & 0,428 & 0,733 & 0,869 & - \\
\hline $100 \%$ & 0,001 & 0,001 & 0,001 & 0,002 & 0,002 & 0,330 & 0,600 & 0,727 & 0,853 \\
\hline
\end{tabular}

It was observed that in primary stimulus that has values of background of $50 \%$ and $60 \%$ RTV, the effect was the weakest precisely because the values of the secondary stimulus are approximately similar to the primary stimulus. Primary stimulus that has values of the secondary stimulus from $70 \%, 80 \%, 90 \%$ and $100 \%$ RTV, the effect is the strongest because the values of the secondary stimuli are darker than of the primary stimuli, which is stated by the definition of the simultaneous contrast effect itself. The most significant differences were observed in a combination where primary stimuli had the values of secondary stimuli of $80 \%, 90 \%$ and $100 \%$ RTV and which have noticeably significant deviations from other primary stimuli. In the combinations of the primary and secondary stimuli, respondents had large deviations when tuning the test and referential primary stimuli.

\section{CONCLUSION}

The research from this work shows obtained results of visual effect of induction and its occurrence on a particular graphic medium. By means of the statistical data analysis, the numerical intensity and direction of lightness deviation among physical and perceived value of primary stimuli of $50 \%$ RTV are clearly shown, depending on the change in the value of the secondary stimuli from $10 \%$ RTV to $100 \%$ RTV.

In the primary stimuli of $50 \%$ RTV with the same value of the secondary stimuli, the slightest deviation in lightness was observed precisely because the primary and secondary stimuli have identical RTV values, which is why it was the easiest for the respondents to tune that value of the primary stimuli. The perceived lightness of the primary and secondary stimuli in that case merged, i.e. the effect of assimilation occurred. The highest intensity of the effect was observed in primary stimuli which has value of the $100 \%$ RTV background. In general, a higher intensity of the effect was observed in primary stimuli with higher values of the secondary stimuli (70\% RTV - 100\% RTV), while a lower intensity of the effect $(10 \%$ RTV - $40 \%$ RTV) was observed in the primary stimuli that had a smaller value of background. In a certain number of cases, the researched effect occurs in the printing process due to specifically constructed conceptual solution that has a negative repercussion when transferring desired message from one reproduction system to another. The researched effect almost always occurs if there is a print on the margin and if banners appear on the edges of a page in a printed product.

The conducted research indicates the imperfections of the human visual system, which can be seen in a concrete example in which the respondents had the task of tuning the appearance of the primary stimuli on a reproduction produced with the help of the CIP3 system and of the primary stimuli on a reproduction that they themselves tuned so that it looked as the "referential" one. The model from this research can be used to provide effective predictions of the occurrence of the simultaneous contrast effect, as well as its precise calculation of intensity under the aforementioned production conditions, set forth in this paper, all with the aim of constructing a conceptual solution in which occurrence of researched effect is reduced to a minimum.

\section{REFERENCES}

[1] Von Goethe, J. W. (2015). Zur Farbenlehre. Jazzybee Verlag.

[2] Chevreul, M. E. (1839). De la loi de contrastesimultané des couleurset de ses applications.

[3] Takasaki, H. (1967). Chromatic Changes Induced by Changes in Chromaticity of Background of Constant Lightness. Journal of the Optical Society of America, 57(1), 93-96. https://doi.org/10.1364/JOSA.57.000093

[4] Takasaki, H. (1966). Lightness Change of Grays Induced by Change in Reflectance of Gray Background*. Journal of the Optical Society of America, 56(4), 504-509. https://doi.org/10.1364/JOSA.56.000504

[5] Logvinenko, A. D. (2002). The anchoring effect in lightness perception in humans. Neuroscience Letters, 334(1), 5-8. https://doi.org/10.1016/S0304-3940(02)00935-7

[6] Fairchild, M. D. (2013). Color Appearance Models: Fairchild/Color Appearance Models. Chichester, UK: John Wiley \& Sons, Ltd. https://doi.org/10.1002/9781118653128

[7] Cernea, P. (2002). The simultaneous contrast of the colors in Van Gogh paints. Oftalmologia (Bucharest, Romania: 1990), 55(4), 96-100.

[8] Milković, M., Mrvac, N., \& Vusić, D. (2009). Vizualna psihofizika i dizajn. Veleučilište u Varaždinu, Varaždin.

[9] Chen, A., Dinet, E., \&Hardeberg, J. Y. (2013). The creation of an artwork with simultaneous contrast. Unpublished. https://doi.org/10.13140/rg.2.1.4400.1684 
[10] Gonzalez, R., \& Powers, A. S. (1973). Simultaneous contrast in goldfish. Animal Learning \& Behavior, 1(2), 96-98. https://doi.org/10.3758/BF03214570

[11] Economou, E., Zdravkovic, S., \& Gilchrist, A. (2007). Anchoring versus spatial filtering accounts of simultaneous lightness contrast. Journal of Vision, 7(12), 1-15. https://doi.org/10.1167/7.12.2

[12] Bergstrom, S. S. (1977). Common and relative components of reflected light as information about the illumination, colour, and three-dimensional form of objects. Scandinavian Journal of Psychology, 18(1), 180-186. https://doi.org/10.1111/j.1467-9450.1977.tb00275.x

[13] Gilchrist, A. L. (1979). The Perception of Surface Blacks and Whites. Scientific American, 240(3), 112-125. https://doi.org/10.1038/scientificamerican0379-112

[14] McNamara, A. (2001). Visual Perception in Realistic Image Synthesis. Computer Graphics Forum, 20(4), 211-224. https://doi.org/10.1111/1467-8659.00550

[15] Kjelldahl, L. \& Schenkman, B. N. (2007). Colour induction on computer displays - adjacency and shape effects. Behaviour \& Information Technology, 26(3), 261-272. https://doi.org/10.1080/01449290500402429

[16] Wu, R.-C., Wardman, R. H., \& Luo, M. R. (2005). A comparison of lightness contrast effects in CRT and surface colours. Color Research \& Application, 30(1), 13-20. https://doi.org/10.1002/col.20074

[17] Soranzo, A., \& Agostini, T. (2004). Impossible Shadows and Lightness Constancy. Perception, 33(11), 1359-1368. https://doi.org/10.1068/p5282

[18] Ron, E. \& Spitzer, H. (2011). Is the Kanizsa illusion triggered by the simultaneous contrast mechanism? Journal of the Optical Society of America A, 28(12), 2629-2641. https://doi.org/10.1364/JOSAA.28.002629

[19] Vladusich, T. (2012). Simultaneous contrast and gamut relativity in achromatic color perception. Vision Research, 69, 49-63. https://doi.org/10.1016/j.visres.2012.07.022

[20] Zavagno, D., Daneyko, O., \& Liu, Z. (2018). The Influence of Physical Illumination on Lightness Perception in Simultaneous Contrast Displays. i-Perception, 9(4), 1-22. https://doi.org/10.1177/2041669518787212

[21] Soranzo, A., Lugrin, J.-L., \& Wilson, C. J. (2013). The effects of belongingness on the Simultaneous Lightness Contrast: A virtual reality study. Vision Research, 86, 97106. https://doi.org/10.1016/j.visres.2013.04.012

[22] Menshikova, G. Y. (2013). An investigation of 3D images of the simultaneous-lightness-contrast illusion using a virtualreality technique. Psychology in Russia: State of Art, 6(3), 49-59. https://doi.org/10.11621/pir.2013.0305

[23] Hajdek, K., Budimir, I., \& Milković, M. (2016). Difference in perceived lightness at achromatic reproductions conditioned with manifestation of the simultaneous contrast effect. Presented at the International conference of printing, design and graphic communication Blaž Baromić 2016.

[24] Hajdek, K., Budimir, I., \& Krizmanić, K. (2016). The appearance of the visual effect simultaneous contrast depending on the printing substrate. Acta Graphica, 27(3), $7-14$.

[25] Hajdek, K., Budimir, I., Matijević, M., \& Mikota, M. (2019). A shift in the perception of lightness on image in digital media due to the manifestation of the simultaneous contrast effect. The Imaging Science Journal, 67(2), 115-122. https://doi.org/10.1080/13682199.2019.1565394

[26] Hajdek, K., Budimir, I., \& Vusić, D. (2016). The intensity of the simultaneous contrast effect depending on the change of background lightness. Tehnički vjesnik, 23(2), 525-531. https://doi.org/10.17559/TV-20160122205606

[27] Fechner, G. (1966). Elements of psychophysics. Vol. I.
[28] Goldstein, E. B. (Ed.). (2005). Blackwell Handbook of Sensation and Perception. Malden, MA, USA: Blackwell Publishing Ltd. https://doi.org/10.1002/9780470753477

[29] Braun, K. M., Fairchild, M. D., \& Alessi, P. J. (1996). Viewing techniques for cross-media image comparisons. Color Research \& Application, 21(1), 6-17. https://doi.org/10.1002/(SICI)1520-6378(199602)21:1<6::AIDCOL1>3.0.CO;2-\#

\section{Contact information:}

Martina HAJDEK, MSc

Faculty of Graphic Arts

Getaldićeva ulica 2, 10000 Zagreb, Croatia

martina.hajdek@grf.hr

Krunoslav HAJDEK, Assis. Prof., PhD

(Corresponding author)

University North

Trg dr. Žarka Dolinara 1, 48000 Koprivnica, Croatia

khajdek@unin.hr

Ivan BUDIMIR, Assis. Prof., PhD

Faculty of Graphic Arts

Getaldićeva ulica 2, 10000 Zagreb, Croatia

ivan.budimir@grf.hr 